Target Diagnostic Operations Summary August 28-29, 2009

D. W. Larson

September 4, 2009 
This document was prepared as an account of work sponsored by an agency of the United States government. Neither the United States government nor Lawrence Livermore National Security, LLC, nor any of their employees makes any warranty, expressed or implied, or assumes any legal liability or responsibility for the accuracy, completeness, or usefulness of any information, apparatus, product, or process disclosed, or represents that its use would not infringe privately owned rights. Reference herein to any specific commercial product, process, or service by trade name, trademark, manufacturer, or otherwise does not necessarily constitute or imply its endorsement, recommendation, or favoring by the United States government or Lawrence Livermore National Security, LLC. The views and opinions of authors expressed herein do not necessarily state or reflect those of the United States government or Lawrence Livermore National Security, LLC, and shall not be used for advertising or product endorsement purposes.

This work performed under the auspices of the U.S. Department of Energy by Lawrence Livermore National Laboratory under Contract DE-AC52-07NA27344. 


\section{Target Diagnostic Operations Summary August 28-29, 2009}

\section{Experiment: Hohlraum_Act_D_S08d}

This campaign will validate the capability of fielding cryogenic hohlraums by demonstrating that the radiation temperature is not affected by contamination and ice buildup.

First NIF shot with cryogenically cooled target.

19K 0.9 scale Au HVac target. 600 kJ with 2ns FIT pulse, 192 beams with Rev 1 and Rev 3 CPPs.

\section{Shot ID: N090828-002-999}

\section{Issues / Results Summary}

Good data acquired on all participating diagnostics.

This was the first shot with NToF participating. Since no neutrons are generated by this shot, the purpose of participating NToF was to find out how much unwanted signal is generated by the Xrays and EMI. The interesting traces produced by this shot are included at the end of this report.

\section{Known Issues, still with us:}

\section{FABS}

Randomly at any time, a GSCP Camera "CCD Initialized" status will go red. Have to command Re-Init CCD, then all is well again. Existing Problem Log 294562.

\section{NBI}

NBI Q31B SRS camera cooler does not work - creates alarms. Existing Problem Log \#294565.

NBI Q31B SRS camera cooler problem exposes an error in the TDS shot software: if a diagnostic has an error so that the rolled-up Ready status is "Usable" instead of "Ready", then the shot software does not handle the situation correctly. The shot software does not wait for all the participating diagnostics to get to the Set state before figuring the Min Tick, so Min Tick is set incorrectly. Until the software is fixed, the workaround is to set the Shot Supervisor to Manual Mode so the Min Tick step may be initiated after all the diagnostics are in the Set state. New Problem Log \#295388.

Shot software tries to send GoTo setpoint commands to non-existent NBI SPECTRALON-CCD cameras. Result is all Macrosteps that setup the video cameras fail. Existing Problem Log \#294566. 


\section{Operators}

\section{TDOs}

Jeff Baron

Brandi Lechleiter

\section{TDC}

Karl Pletcher

\section{Participation}

SXI-Lower (161,326)
SXI-Upper $(018,123)$
Dante-Lower (143-274)
Channels 1-18
Dante-Upper (064-350)
FABS Q31B
Camera B315
Camera B316
Camera B317
Camera B318
SRS Fast Diode Scope
SBS Fast Diode Scope
Slow Diode Scope
Streak Camera SRS
Streak Camera SBS
Cal Spect Camera
Gate Monitor Scope
FABS Q36B
Camera B365
Camera B366
Camera B367
Camera B368
SRS Fast Diode Scope
SBS Fast Diode Scope
Slow Diode Scope
Streak Camera SRS
Streak Camera SBS
Cal Spect Camera
Gate Monitor Scope
NBI (Q31B)
Scope

\section{Secondary}

Dropped (Camera replaced with an 'image plate', which is completely passive, so no software control required or desired)

Primary

Primary

Dropped

Secondary

Secondary

Secondary

Secondary

Secondary

Secondary

Secondary

Secondary

Secondary

Secondary

Secondary

Secondary

Secondary

Secondary

Secondary

Secondary

Secondary

Secondary

Secondary

Secondary

Secondary

Secondary

Secondary

Secondary

Secondary

Secondary 


$\begin{array}{lc}\text { GSCP } & \text { Not Used } \\ \text { SRS Camera } & \text { Secondary } \\ \text { SRS Diode } & \text { Secondary } \\ \text { SBS Camera } & \text { Secondary } \\ \text { SBS Diode } & \text { Secondary } \\ \text { BI (Q36B) } & \text { Secondary } \\ \text { Scope } & \text { Secondary } \\ \text { GSCP } & \text { Not Used } \\ \text { SRS Camera } & \text { Secondary } \\ \text { SRS Diode } & \text { Secondary } \\ \text { SBS Camera } & \text { Secondary } \\ \text { SBS Diode } & \text { Secondary } \\ \text { PMTs 1-10 } & \text { Secondary } \\ \text { EMP } & \text { Secondary } \\ \text { ToF 4m }(064,275) & \text { Tertiary, run manually } \\ \end{array}$

\section{Setup}

\section{Timing}

\section{Client Delays}

\section{SXI-Lower $(161,326)$}

CCD -Trigger (CCD) 500,000,000 ns

Dante-1 (Lower, 143,274)

$\begin{array}{ll}\text { Trigger } 1 & 0.0 \mathrm{~ns}\end{array}$

Trigger $2 \quad 9.0 \mathrm{~ns}$

Trigger $3 \quad 4.5 \mathrm{~ns}$

\section{FABS Q31B}

CAL-SPECT-CCD $\quad-890 \mathrm{~ns}$ (was changed in database today from $-894 \mathrm{~ns}$ )

SBS Streak CCD 500,000,000 ns

SBS Streak Comb $\quad-1234$ ns

SBS Streak Sweep $\quad-284.637 \mathrm{~ns}$

SCOPE $0 \mathrm{~ns}$

SPECTRALON-CCD $\quad-750,000 \mathrm{~ns}$

SRS Streak CCD 500,000,000 ns

SRS Streak Comb $\quad-1385 \mathrm{~ns}$

SRS Streak Sweep $\quad-424.773$ ns

\section{FABS Q36B}

CAL-SPECT-CCD $\quad-700.0 \mathrm{~ns}$ setpoints have three different values, is -700 for Shot SBS Streak CCD 500,000,000 ns

SBS Streak Comb $\quad-1100 \mathrm{~ns}$ 


$\begin{array}{ll}\text { SBS Streak Sweep } & -194 \mathrm{~ns} \quad \text { (was changed in database today from - } 184 \mathrm{~ns} \text { ) } \\ \text { SCOPE } & 0 \mathrm{~ns} \\ \text { SPECTRALON-CCD } & -750,000 \mathrm{~ns} \\ \text { SRS Streak Comb } & -1150 \mathrm{~ns} \\ \text { SRS Streak Sweep } & -197 \mathrm{~ns} \\ \text { SRS Streak CCD } & 500,000,000 \mathrm{~ns}\end{array}$

\section{NBI (Q31B)}

Cal Laser DIAG $\quad-1093$ ns

\section{NBI (Q36B)}

Cal Laser DIAG $\quad-1093$ ns

\section{FFLEX}

Trigger

$412 \mathrm{~ns}$

\section{$\underline{E E M P}$}

Scopes

$345,345,345 \mathrm{~ns}$

\section{NToF 4m $(064,275)$}

Scope Trigger

0 ns

\section{Configurations}

\section{Dante-Lower $(143,274)$}

Attenuator configuration was not finalized in time to put into M-SSAR. Verified configuration per setup sheet from Dante RI.

\begin{tabular}{|c|l|}
\hline Channel & $\begin{array}{c}\text { Current } \\
\text { attenuators }(\mathrm{dB})\end{array}$ \\
\hline 1 & 6,6 \\
\hline 2 & 6,20 \\
\hline 3 & 14,14 \\
\hline 4 & $2,2,14$ \\
\hline 5 & $20,2,2$ \\
\hline 6 & 6,14 \\
\hline 7 & 10,14 \\
\hline 8 & 6,20 \\
\hline 9 & $1,3,20$ \\
\hline 10 & 10,20 \\
\hline 11 & 20,14 \\
\hline 12 & $6,6,6$ \\
\hline
\end{tabular}




\begin{tabular}{|l|l|}
\hline 13 & 14 \\
\hline 14 & 2,6 \\
\hline 15 & 20,20 \\
\hline 16 & 20,20 \\
\hline 17 & 20,20 \\
\hline 18 & 20,20 \\
\hline
\end{tabular}

\section{FABS Q31B}

\section{SRS Fast Diode Scope}

Channel 1 Vertical Range $2 \mathrm{~V}$

Channel 2 Vertical Range $2 \mathrm{~V}$

Channel 3 Vertical Range $2 \mathrm{~V}$

Channel 4 Vertical Range $2 \mathrm{~V}$

\section{SBS Fast Diode Scope}

Channel 1 Vertical Range $2 \mathrm{~V}$

Channel 2 Vertical Range $2 \mathrm{~V}$

Channel 3 Vertical Range $2 \mathrm{~V}$

Channel 4 Vertical Range 2 V

\section{Slow Diode Scope}

Channel 1 Vertical Range $2 \mathrm{~V}$

Channel 2 Vertical Range $2 \mathrm{~V}$

Channel 3 Vertical Range $2 \mathrm{~V}$

Channel 4 Vertical Range 2 V

\section{SRS Streak Camera}

Sweep Speed 40ns

\section{SBS Streak Camera}

Sweep Speed 40ns

\section{FABS Q36B}

\section{SRS Fast Diode Scope}

Channel 1 Vertical Range $2 \mathrm{~V}$

Channel 2 Vertical Range $2 \mathrm{~V}$

Channel 3 Vertical Range $2 \mathrm{~V}$

Channel 4 Vertical Range $2 \mathrm{~V}$

\section{SBS Fast Diode Scope}

Channel 1 Vertical Range 2 V

Channel 2 Vertical Range 2 V

Channel 3 Vertical Range 2 V 
Channel 4 Vertical Range 2 V

\section{Slow Diode Scope}

Channel 1 Vertical Range 2 V

Channel 2 Vertical Range $2 \mathrm{~V}$

Channel 3 Vertical Range 2 V

Channel 4 Vertical Range 2 V

\section{SRS Streak Camera}

Sweep Speed

\section{SBS Streak Camera}

Sweep Speed

\section{NBI Q31B}

\section{Scope}

Channel 1 Vertical Scale

Channel 2 Vertical Scale

Channel 3 Vertical Scale

Channel 4 Vertical Scale
$0.1 \mathrm{~V} /$ division

$1.0 \mathrm{~V} /$ division

$1.0 \mathrm{~V} /$ division

$1.0 \mathrm{~V} /$ division

\section{NBI Q36B}

\section{Scope}

Channel 1 Vertical Scale Channel 2 Vertical Scale Channel 3 Vertical Scale Channel 4 Vertical Scale
$0.1 \mathrm{~V} /$ division $1.0 \mathrm{~V} /$ division $1.0 \mathrm{~V} /$ division $1.0 \mathrm{~V} /$ division

\section{NToF 4m $(064,275)$}

\section{Scope}

Channel 1 Vertical Scale $0.05 \mathrm{~V} /$ division Channel 2 Vertical Scale $0.01 \mathrm{~V} /$ division

Channel 3 Vertical Scale $0.01 \mathrm{~V} /$ division Channel 4 Vertical Scale $0.1 \mathrm{~V} /$ division 
FLEX Settings

\begin{tabular}{|c|c|c|c|c|c|c|}
\hline Scope & $\begin{array}{c}\text { Horizontal } \\
\text { Scale } \\
{[\mu \mathrm{s} / \mathrm{div}]} \\
\end{array}$ & $\begin{array}{c}\text { PM } \\
\text { Tube } \\
\# \\
\end{array}$ & $\begin{array}{c}\text { HVPS } \\
\text { Voltage } \\
{[\mathrm{V}]} \\
\end{array}$ & $\begin{array}{c}\text { Capacitance } \\
{[\mathrm{pF}]}\end{array}$ & $\begin{array}{c}\text { Channel } \\
\#\end{array}$ & $\begin{array}{c}\text { Vertical } \\
\text { Full Range } \\
{[\text { [V] }} \\
\end{array}$ \\
\hline \multirow{4}{*}{1} & \multirow{4}{*}{25} & \multirow{2}{*}{1} & \multirow{2}{*}{-800} & \multirow{2}{*}{22,000} & 1 & 0.50 \\
\hline & & & & & 2 & 10 \\
\hline & & & & & 3 & 0.50 \\
\hline & & 2 & -830 & 100,000 & 4 & 10 \\
\hline \multirow{4}{*}{2} & \multirow{4}{*}{25} & \multirow{2}{*}{3} & \multirow{2}{*}{-800} & \multirow{2}{*}{100,000} & 1 & 0.50 \\
\hline & & & & & 2 & 10 \\
\hline & & \multirow{2}{*}{4} & \multirow{2}{*}{-820} & \multirow{2}{*}{100,000} & 3 & 0.50 \\
\hline & & & & & 4 & 10 \\
\hline \multirow{4}{*}{3} & \multirow{4}{*}{25} & \multirow{2}{*}{5} & \multirow{2}{*}{-900} & \multirow{2}{*}{22,000} & 1 & 0.50 \\
\hline & & & & & 2 & 10 \\
\hline & & \multirow{2}{*}{6} & \multirow{2}{*}{-730} & \multirow{2}{*}{22,000} & 3 & 0.50 \\
\hline & & & & & 4 & 10 \\
\hline \multirow{4}{*}{4} & \multirow{4}{*}{25} & \multirow{2}{*}{7} & \multirow{2}{*}{-760} & \multirow{2}{*}{22,000} & 1 & 0.50 \\
\hline & & & & & 2 & 10 \\
\hline & & \multirow{2}{*}{8} & \multirow{2}{*}{-870} & \multirow{2}{*}{22,000} & 3 & 0.50 \\
\hline & & & & & 4 & 10 \\
\hline \multirow{4}{*}{5} & \multirow{4}{*}{25} & \multirow{2}{*}{9} & \multirow{2}{*}{$1,100 \dagger$} & 4700 & 1 & 0.50 \\
\hline & & & & $4, / 00$ & 2 & 10 \\
\hline & & 10 & $1400 *$ & 1700 & 3 & 0.50 \\
\hline & & 10 & $1,400 \uparrow$ & $4, / 00$ & 4 & 10 \\
\hline & & 0 & $1100 *$ & & 1 & 0.50 \\
\hline 6 & 05 & 9 & 1,1001 & & 2 & 10 \\
\hline 0 & 0.5 & 10 & $1.400 \%$ & & 3 & 0.50 \\
\hline & & 10 & $1,400 \uparrow$ & & 4 & 10 \\
\hline
\end{tabular}

$\uparrow$ Not listed in M-SSAR.

EEMP

\begin{tabular}{|r|c|c|c|}
\cline { 2 - 4 } \multicolumn{1}{c|}{} & Scope 1 & Scope 2 & Scope 3 \\
\hline Channel 1 V/div & 0.5 & 0.5 & 0.5 \\
\hline Channel 2 V/div & 1.0 & 0.5 & 0.5 \\
\hline Channel 3 V/div & 0.5 & 0.5 & 0.5 \\
\hline Channel 4 V/div & 1.0 & 0.1 & 0.5 \\
\hline Sweep Speed, ns/div & 200 & 200 & 200 \\
\hline
\end{tabular}

\section{Startup Issues}

Dante Scope 18 reported IO_ERROR. Investigation found that the GPIB address selector switches had been disturbed. Set them all to zero, then to desired address. Now works OK. 


\section{Independent Dry Runs}

All OK.

\section{Integrated Dry Run \#1}

Had a variety of odd problems, so we decided to try again.

\section{Integrated Dry Run \#2}

No alarms. All looked good.

\section{Rod Shot N090828-002-001}

All looked good, but TDS set Min Tick to -120 , should be something more like -250 . This is a known issue, we just had to remember what to do about it.

\section{Rod Shot N090828-002-002}

Ran Shot Supervisor in Manual Mode and Min Tick gets figured correctly.

Shot aborted due to MOR problem.

\section{Rod Shot N090828-002-003}

Shot aborted due to PEPC problem.

\section{Rod Shot N090828-002-004}

Except for two nuisance alarms, all looked good.

\section{FFLEX (090,110) HVPS}

When going to Set state, got this nuisance alarm:

OUT OF RANGE - /PS350@GPIB0::1::INSTR/SetPoints/VoltageSetting, current value = -

800.0. Target:-1250.0 RangeType:TargetExactVoltage Setting Range condition

Controller GUI indicated the voltage was set as desired.

\section{FABS Q36B SBS Streak Camera}

At about $\mathrm{t}+20$, got this nuisance alarm:

OUT OF RANGE - SiCcd@0/CCD Trigger State, current value = TRIGGERED. Target:IDLE RangeType:TargetExact CCD Trigger State range condition

\section{System Shot N090828-002-999}

Shot fired at 2009 August 29 02:48:36 


\section{SXI Lower (161,326)}

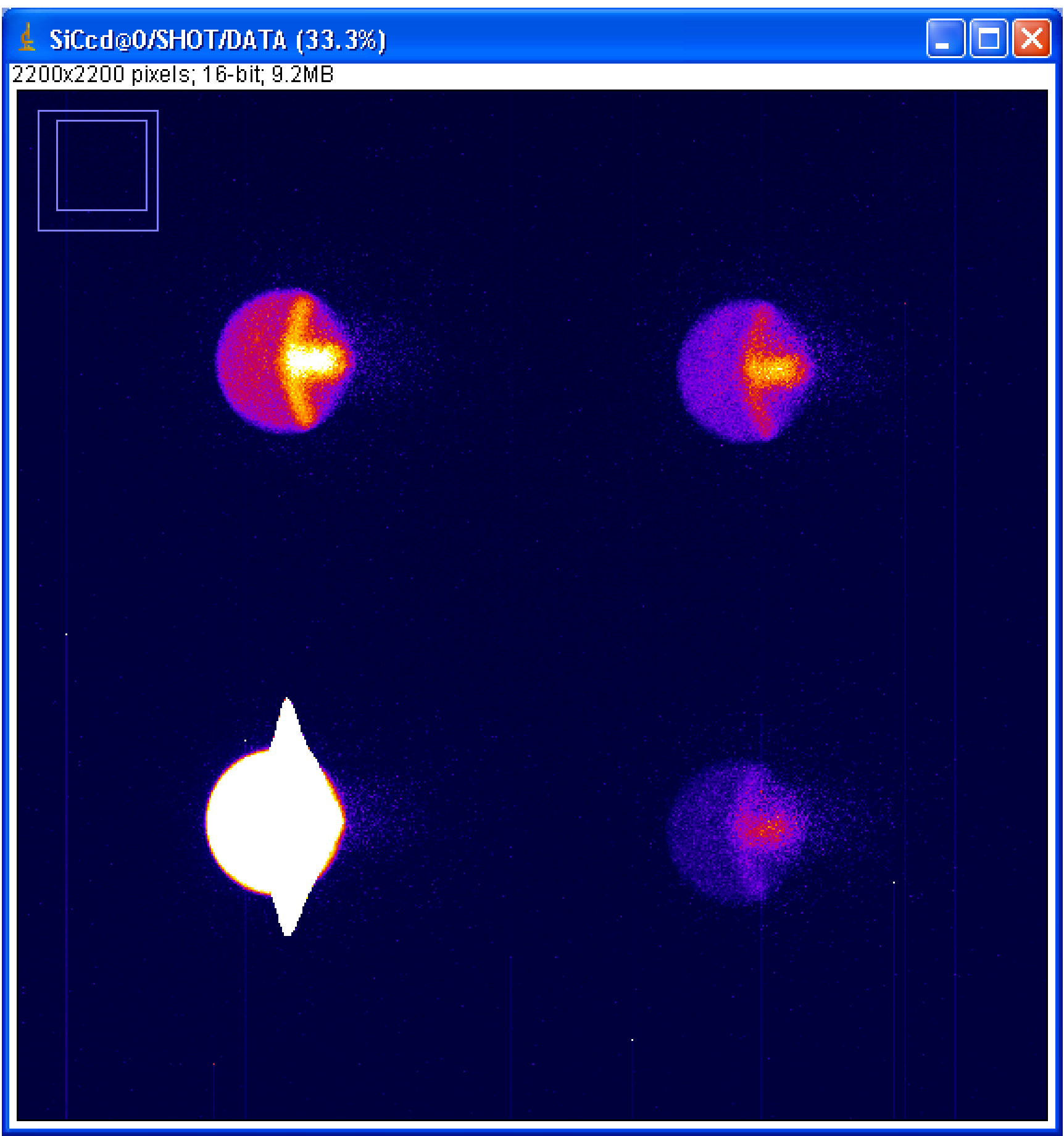

\section{Dante-1 (143,274)}

All channels recorded high quality pulses.

At about $\mathrm{t}+40$ got this alarm from the HVPS:

OUT OF RANGE - /PS350@GPIB0::1::INSTR/Status/CurrentTripEvent, current value = true. 
Target:false RangeType:TargetExactCurrent Trip Event condition.

Controller GUI also indicates Current Trip.

\section{FABS Q31B}

No problems. All data acquired.

\section{B315 Spectralon CCD}

\section{FABS Q36B}

Good data acquired.

During countdown, SRS Fast Diode Scope indicated Triggered on prematurely on TD Shot Manager GUI, but controller GUI indicated armed. This is a known issue.

\section{NBI Q31B}

No problems. All data acquired. 


\section{SBS}

d Pimar(Go/Images/SHOT/DATA (50\%)

-

$1024 \times 1024$ pixels; 32 -bit; $4 \mathrm{MB}$

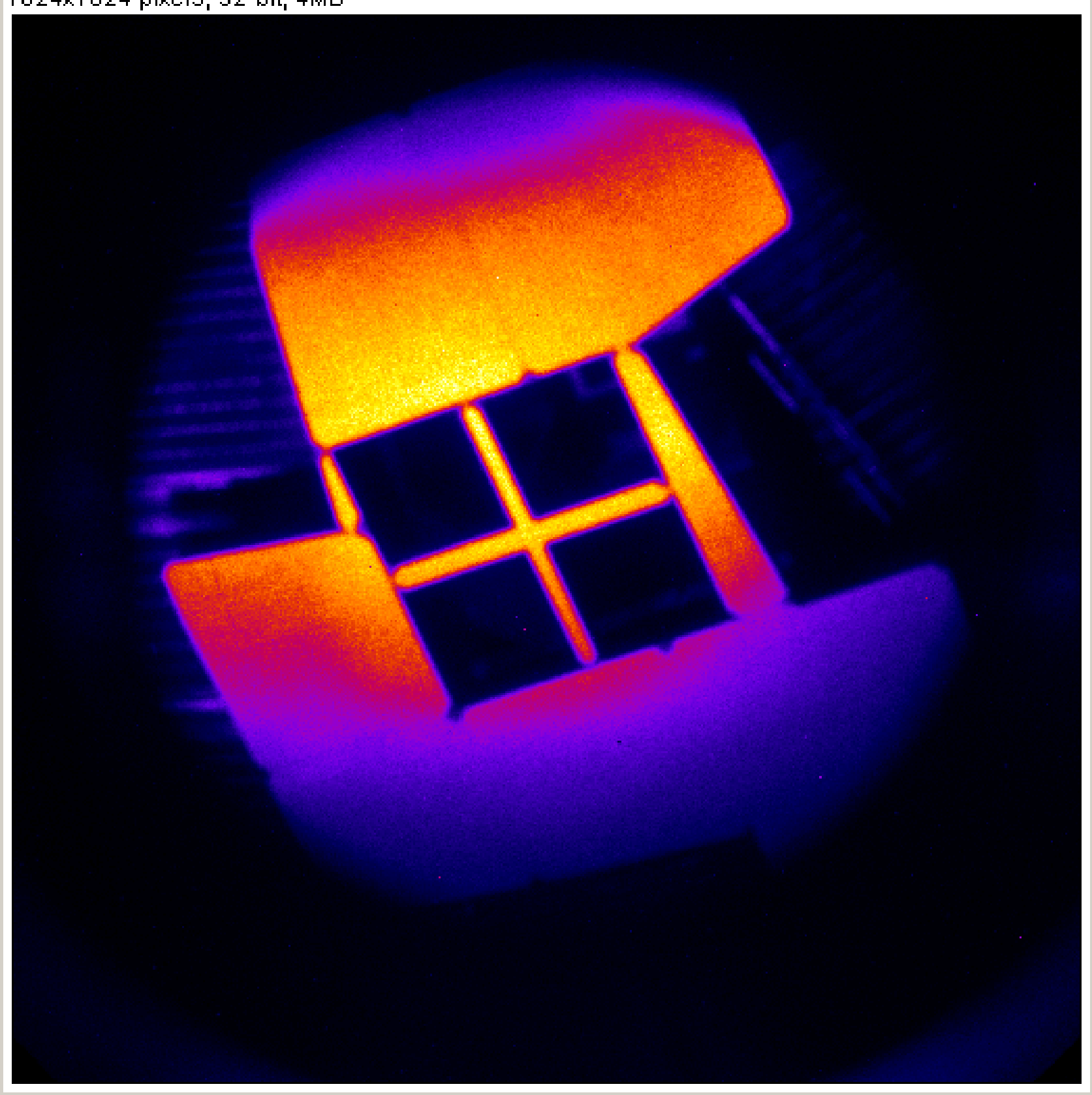

page 11 of 15 


\section{NBI Q36B}

No problems. All data acquired.

\section{SRS}

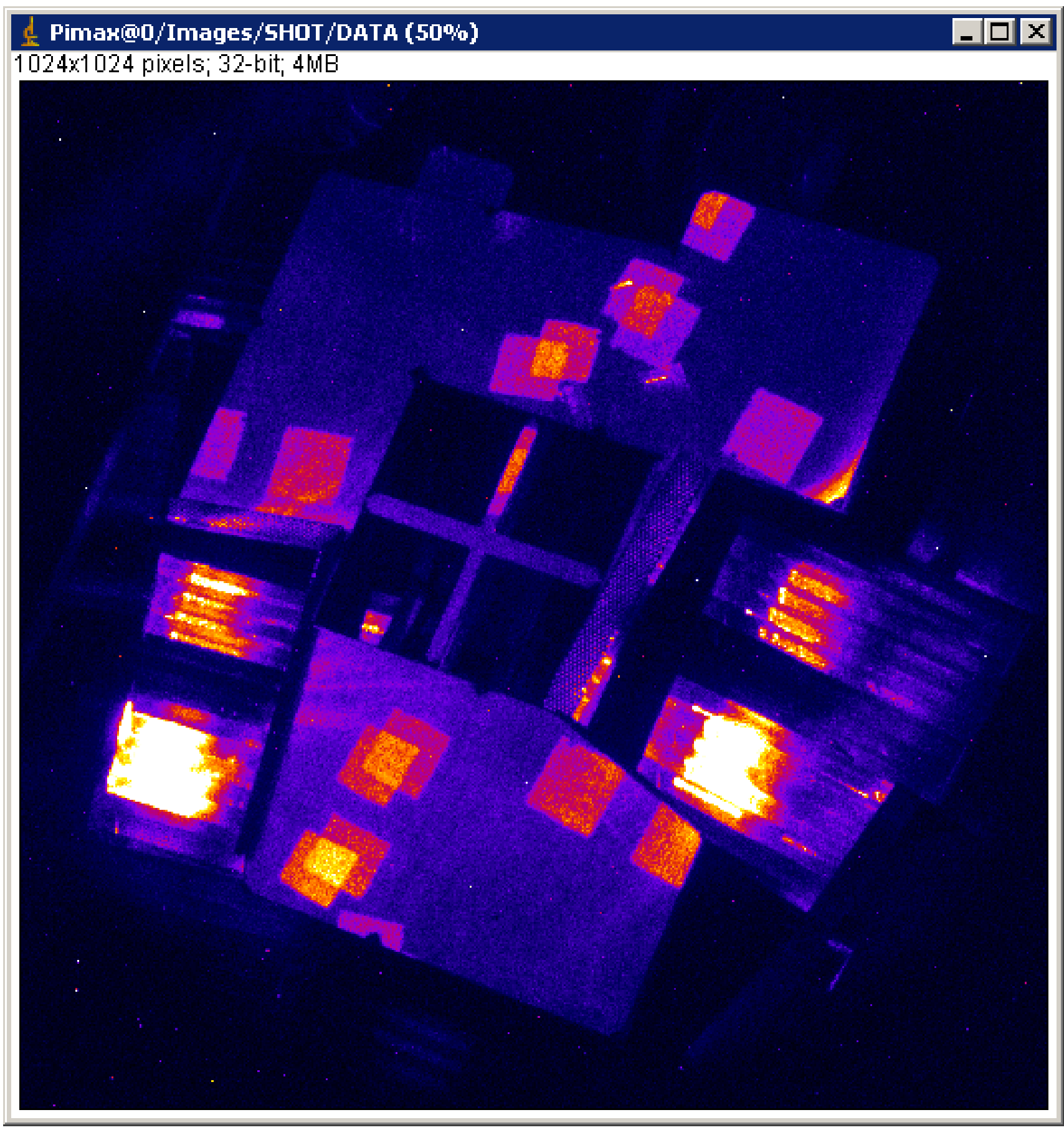




\section{FFLEX (090,110)}

Recorded good pulses. Only clipped pulse was on Scope 3 channel 1.

Going to Set state, got a couple of the nuisance alarms from two of the HVPSs, similar to that obtained during Rod Shot

During countdown, FFLEX scope 6 indicated Triggered prematurely on TD Shot Manager GUI, but controller GUI indicated armed. This is a known issue.

\section{EEMP}
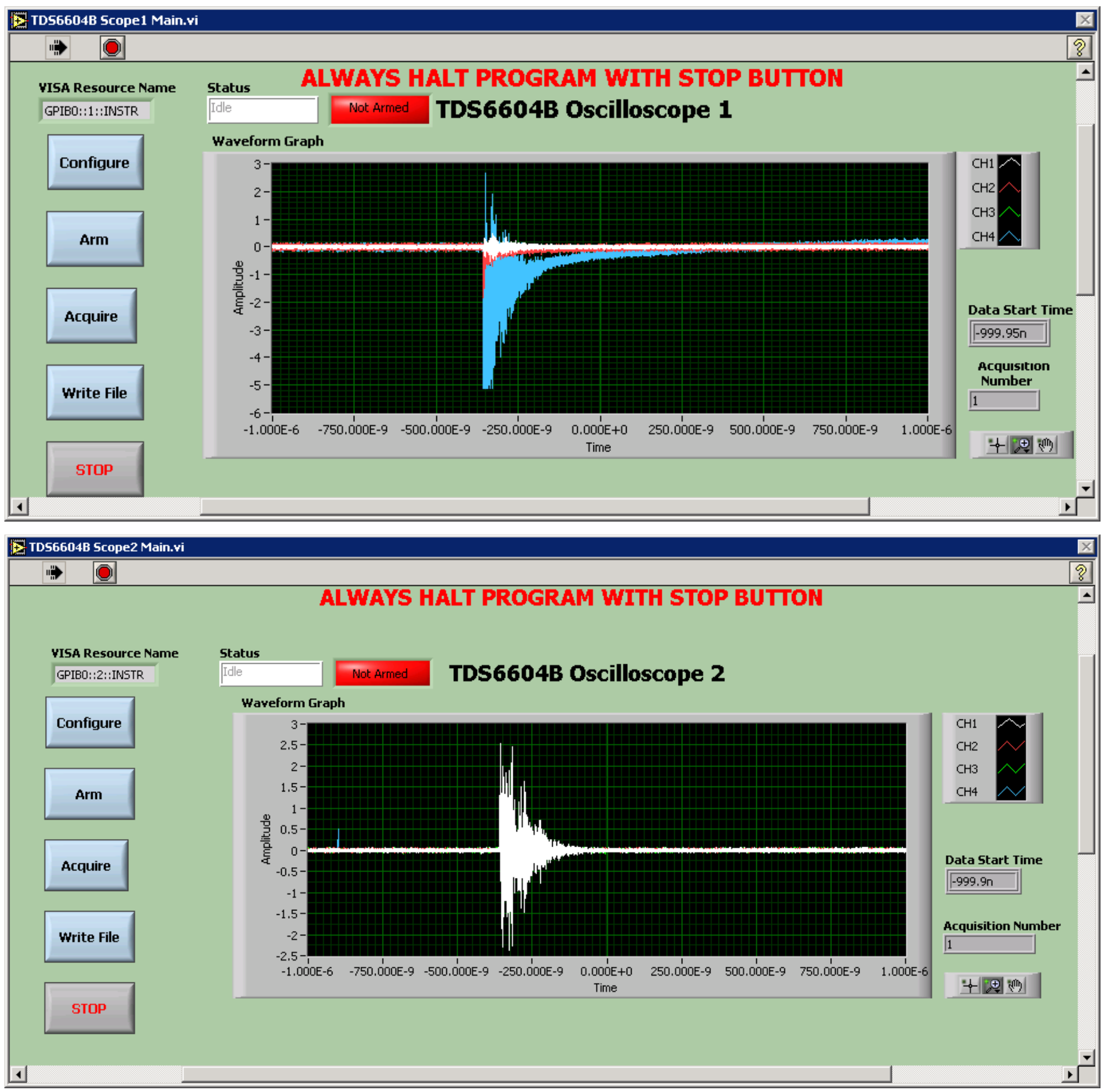


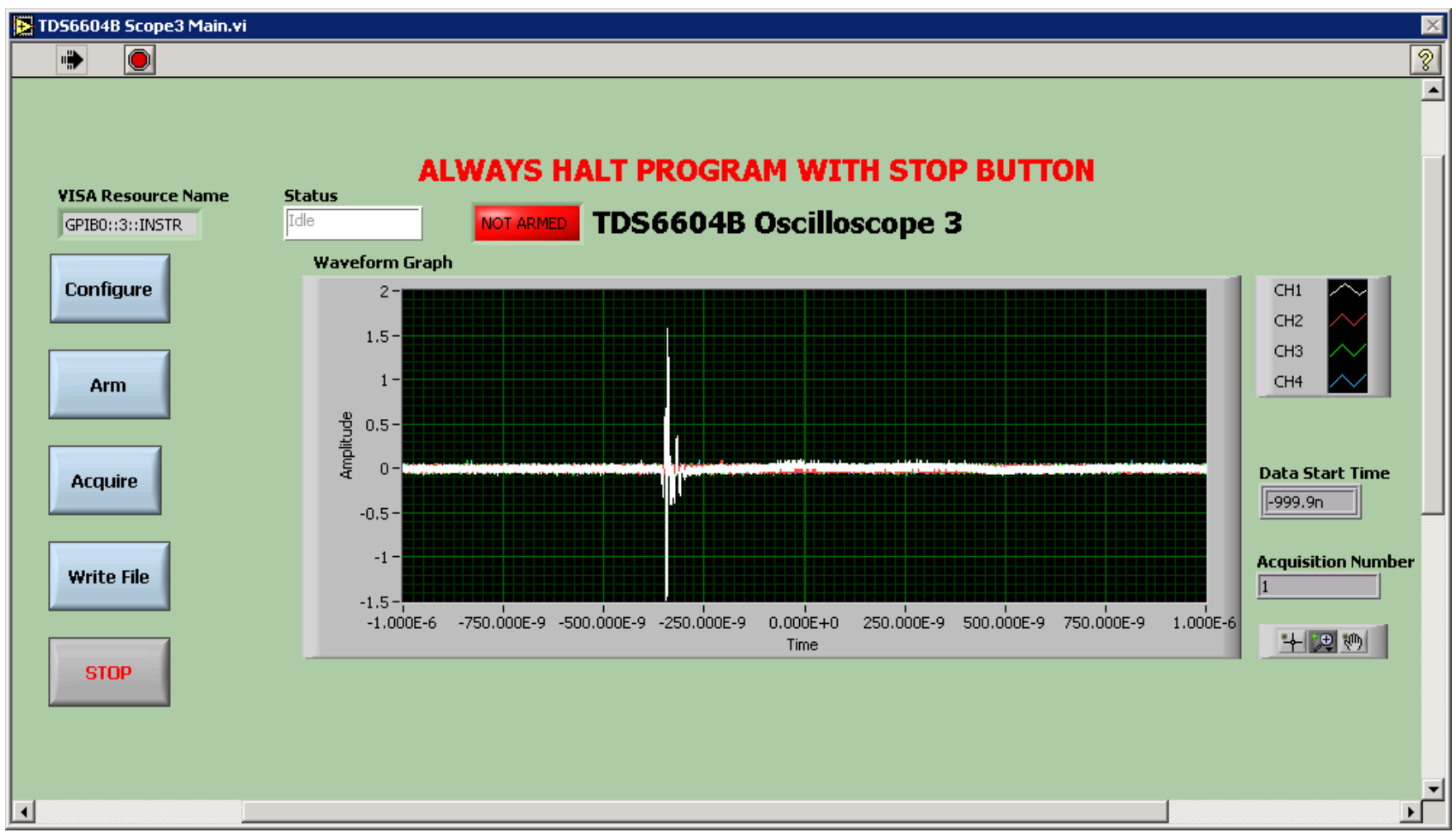

page 14 of 15 


\section{NToF $4 m(064,275)$}

When going to Set State, got this alarm from TD-NTOF-4M-SC-LCU:

DC Error message: Target_Diag_Lcu.Impl.Concrete.Distribute_Command HTC Disable failed for Unexpected exception in Target Diag_Lcu.Impl.Concrete.Distribute_Command CONSTRAINT_ERROR raised at 16\#01238A84\#, Exception Message: On Trigger TIMING|TC|NTOF-4M|SCOPE-HTRIG; Unexpected exception in

Target_Diag_Lcu.Impl.Concrete.Distribute_Command - CONSTRAINT_ERROR raised at 16\#01238A84\#, Exception Message: On Trigger TIMING|TC|NTOF-4M|GATE-HTRIG

Settings on Trigger GUI look as desired for the shot.

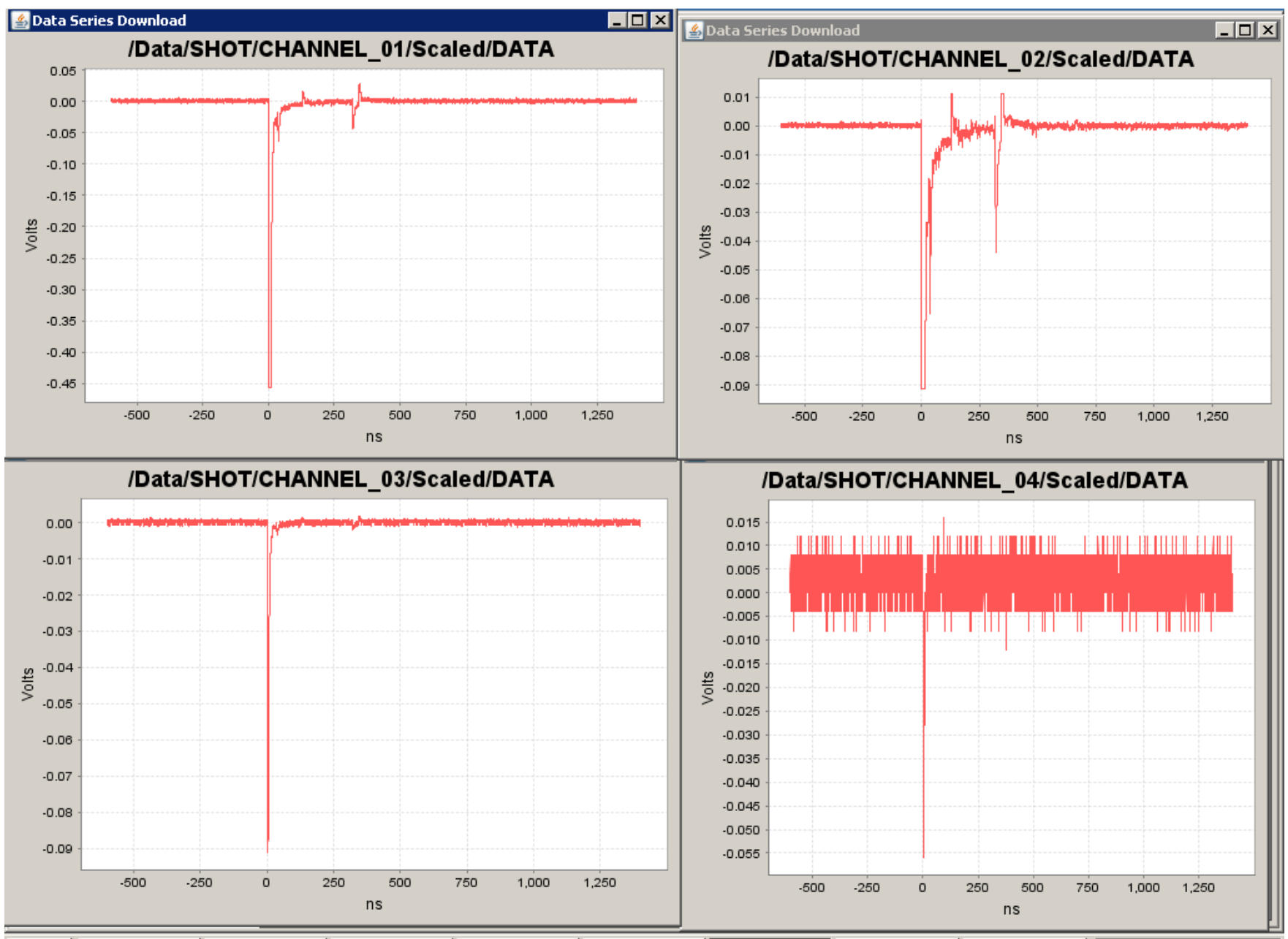

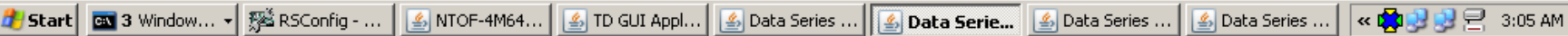

